\title{
Composition en acides aminés et en acides gras d'un aliment de complément à base des ressources alimentaires locales : cas de la Vamine en RD Congo
}

\author{
Gisele Makengo KAFUTI ${ }^{1 *}$, Théophile Mbemba FUNDU ${ }^{2}$ et Marianne SINDIC ${ }^{3}$ \\ ${ }^{1}$ Laboratoire de Biochimie de la Nutrition, Département de Biologie, Faculté des Sciences, Université de \\ Kinshasa, R.D. Congo. \\ ${ }^{2}$ Laboratoire de Biochimie de la Nutrition, Département de Biologie, Faculté des Sciences, Université de \\ Kinshasa, R.D. Congo. \\ ${ }^{3}$ Univ. Liege - Gembloux Agro Bio-Tech. Analysis Quality and Risk Unit, Laboratory of Agro-Food Quality \\ and Safety, Passage des Déportés, 2. B-5030 Gembloux, Belgium. \\ *Auteur correspondant ; E-mail: giselemakengo@yahoo.fr
}

\section{RESUME}

L'objectif de cette étude est de déterminer les teneurs en acides aminés et acides gras d'un aliment de complément, dénommé Vamine, fabriqué à base des produits locaux disponibles et accessibles suivants: voandzou et arachides comme source protéiques; maïs et manioc. La détermination des teneurs en acides aminés essentiels donne, des taux de $115 \mathrm{mg}$ de thréonine; 163,5 mg de valine; 39,25 mg de méthionine; 109,5 $\mathrm{mg}$ de isoleucine; $232,25 \mathrm{mg}$ de leucine; $84,75 \mathrm{mg}$ de Tyrosine; $171 \mathrm{mg}$ de phénylalanine; $149,5 \mathrm{mg}$ de Histidine; $235,25 \mathrm{mg}$ de lysine. Les teneurs en acides gras essentiels sont de $265 \mathrm{mg}$ pour l'acide alpha linolénique et absent pour l'acide linoléique. La comparaison des concentrations des acides aminés et acides gras trouvés avec les normes FAO/OMS montre que les teneurs trouvées sont satisfaisantes et même souvent supérieures aux normes. La Vamine peut devenir un aliment de complément approprié pour la croissance des enfants en République Démocratique du Congo.

(C) 2015 International Formulae Group. All rights reserved.

Mots clés : Produits locaux, Vamine, teneur en acides aminés, teneur en acides gras, aliment de complément, enfant.

\section{INTRODUCTION}

De la naissance à l'âge de 6 mois, tous les besoins nutritionnels de l'enfant sont couverts par le lait maternel (Pronadud, 2014) qui ne peut plus satisfaire les besoins en énergie et en protéines (CP04-01,2004) pendant la période dite de sevrage qui s'étend de 6 mois à 1 an voire 2 ans. En ce moment, il est nécessaire d'apporter des aliments nouveaux, appelés aliment de complément, sous forme liquide ou semi-liquide pour compléter les apports nutritionnels du lait maternel. Ces derniers doivent apporter en proportions équilibrées les protéines, les lipides, les glucides et les sels minéraux (Mouquet-rivier, 2006).

Si l'enfant n'est pas bien nourri, divers signes de malnutrition protéino-énergétique (Bouwer et al., 2000; OPS, 2003) apparaissent rapidement sur son corps. Pour parer à cette 
situation, il est important de disposer, pendant cette période, des farines infantiles de bonne qualité nutritionnelle pour couvrir les besoins nutritionnels de l'enfant.

Cependant, pendant le sevrage, les mères africaines, incapables de se procurer des farines infantiles de bonne qualité importées existant sur le marché à cause de leur coût élevé (Salle, 2009), nourrissent généralement leurs enfants avec des bouillies traditionnelles préparées à partir de farines simples ou composées provenant de céréales, de tubercules, riches en glucides et pauvres en protéines (Briend, 2000).

Dans les pays en développement, les bouillies traditionnelles à base de farines de céréales ou de racines de manioc, étant riches en amidon, elles sont visqueuses, et indigestes; ce qui oblige les mères à y ajouter de l'eau afin de les rendre plus fluides, faciles à avaler et digérer, réduisant de ce fait leur densité énergétique (Pamplona, 2000; Francisca, 2014).

D'où l'autorisation accordée par la FAO/OMS d'adjoindre d'acides aminés isolés aux préparations pour nourrissons afin d'améliorer leur valeur nutritive. Il est par ailleurs important que l'aliment de complément contienne les acides gras insaturés essentiels, acides linoléique, et linoléiques, qui ne peuvent être apportés à l'organisme que par l'alimentation en plus de l'acide arachidonique, à la base de la synthèse des prostaglandines, tromboxannes et des leucotriènes (Mbemba, 2013) C'est pourquoi, depuis quelques années, la FAO/OMS préconisent la fabrication, à base de produits locaux disponibles et accessibles, des aliments de complément de qualité nutritive suffisante, en vue de couvrir les besoins nutritionnels de l'enfant africain (Salle, 2009; Beaufrère et al., 2001; FAO/OMS, 2009).

C'est dans le souci de répondre à cette recommandation que nous nous sommes intéressés à mettre au point un aliment de complément de bonne qualité nutritionnelle, de bonne densité énergétique, et à base de ressources agricoles locales. La présente étude détermine les teneurs en acides aminés et en acides gras de la farine formulée en vue de mieux apprécier la valeur des protéines et la nature des lipides, présents dans la Vamine.

\section{MATERIEL ET METHODES}

\section{Aliments traditionnels utilisés}

L'aliment de complément Vamine a été préparé à partir de tubercules de manioc doux (Manihot esculenta CRANTZ var. dulcis), de graines de Voandzou (Vigna subterranea (L.)Verdc., d'arachides (Arachis hypogea L.), de grains de maïs (Zea mays L.), et de tubercules d'ignames (Dioscorea bulbifera L.).

\section{Production de l'aliment de complément Farine de manioc doux}

Elle est obtenue à partir des tubercules de manioc doux. Après être lavés, épluchés et découpés en petites tranches, ils ont été cuits dans l'eau bouillante pendant 30 minutes. Les tranches cuites ont été séchées à l'étuve à 60 ${ }^{\circ} \mathrm{C}$ pendant 48 heures, pulvérisées au moulin et tamisées.

\section{Farine de maïs grillé}

Elle est obtenue à partir des grains de maïs. Ils ont été triés manuellement, lavés, et ont subi un grillage puis moulus et tamisés.

\section{Farine de voandzou}

Elle est obtenue à partir des grains de voandzou qui ont été triés manuellement, lavés, trempés dans l'eau bouillante pendant 15 minutes, égouttés, et ils ont subi une torréfaction enfin d'être séché à l'étuve à 60 ${ }^{\circ} \mathrm{C}$ pendant une nuit, de pelliculés puis moulus et tamisés.

\section{Pâte d'arachides}

Elle est obtenue à partir des arachides. Ceux-ci ont subi un triage avant d'être grillé; de pelliculés puis moulus pour avoir une pâte.

\section{Formulation de la Vamine}

La farine MAV (maïs, arachides, Voandzou), initialement préparée pour $100 \mathrm{~g}$ de mélange avec de Voandzou, de Manioc, 
d'Arachide et de Maïs, a été modifiée lors de la mise au point des traitements enzymatiques de manière à conserver la valeur énergétique de sa formulation théorique pour $100 \mathrm{~g}$ de Vamine en homogénéisant les farines de Voandzou, de manioc et de maïs; et des pâtes d'arachides à l'aide d'un mélangeur Mx type 676 , puis en y ajoutant à l'aide d'une spatule de FRA, de sucre de canne7,5\%, de sucre vanillé $2,5 \%$ et $1 \%$ de complément minéral (sel iodé $0,5 \%$ et bicarbonate de soude Baking 0,5\%).

\section{Préparation de la farine instantanée de la} Vamine

La préparation de la Vamine instantanée a consisté à mélanger en quantité équivalente la farine Vamine avec l'eau chaude jusqu' à l'obtention d'une pâte élastique. La pâte obtenue a été étalée de façon homogène sur des plateaux puis incubée à $50{ }^{\circ} \mathrm{C}$ pendant 30 minutes à l'incubation WTB Binder. Après 30 minutes d'incubation, la pâte a été cuite au four ménager Electrotech à $130{ }^{\circ} \mathrm{C}$ pendant au moins 45 minutes. La pâte ainsi cuite et sèche a été pulvérisée au moulin pour obtenir la farine précuite qui se dissout instantanément dans l'eau bouillante $\left(100{ }^{\circ} \mathrm{C}\right)$, c'est-à-dire la Vamine instantanée. Nous avons ensuite ajouté du sucre, de l'arôme et un complément minéral dans les proportions appropriées.

\section{Détermination des teneurs en acides gras et en acides aminés \\ Les acides aminés ont été dosés par HPLC (High Performance Liquid} Chromatography) en subissant une hydrolyse acide de leurs protéines. La matière grasse a été dosée par HPLC selon la méthode au BF3/méthanol. La détermination des acides gras a été faite par chromatographie en phase gazeuse selon la méthode au BF3/méthanol donnant des esters méthyliques à partir des acides gras méthanolysés.

BF3 : tri fluorure de Bohr3

\section{RESULTATS}

Détermination des teneurs en acides aminés de la Vamine

La composition en protéines de la Vamine est de 16,3\% par cent grammes de matière sèche. L'analyse des acides aminés contenus dans ces protéines a révélé les résultats repris dans la Figure 1.

Il ressort des résultats obtenus que le glutamate représente environ $23 \%$ de l'ensemble des acides aminés suivis de l'aspartate $(1,41 \%)$, de la leucine $(0,93 \%)$, de la lysine $(0,94 \%)$, de 1 'arginine $(0,91 \%)$, de la serine $(0,72 \%)$, de la phénylalanine $(0,68 \%)$, de la valine $(0,65 \%)$, de l'histidine $(0,6 \%)$, de la glycine $(0,47 \%)$, de la thréonine $(0,46 \%)$ tous les autres acides aminés représentant chacun moins de $1 \%$. La présence de tous les acides aminés a été notée en particulier les acides aminés essentiels, en concentrations appréciables.

\section{Détermination de la teneur en acides gras de la Vamine}

Les résultats des teneurs en acides gras de la Vamine exprimés en pourcentage sont représentés dans la Figure 2. Les données obtenues indiquent que l'acide linolelaidique représente $39,21 \%$, suivi des acides oléique et elaidique $32,43 \%$, de l'acide stéarique $4,88 \%$, de l'acide Béhinique $1,94 \%$, de l'acide arachidonique $1,24 \%$, de l'acide $\alpha$-linolenique $1,06 \%$, d'un deuxième isomère de l'acide oléique, soit l'acide vaccénique $1,04 \%$, de l'acide cis-11-éicosenoique $0,56 \%$ et de l'acide lignocérique $0,51 \%$. L'acide linoléique est en concentration importante mais sous la forme trans (acide linoélaidique) à l'HPLC, de même que les acides arachidonique et alpha linolénique. 


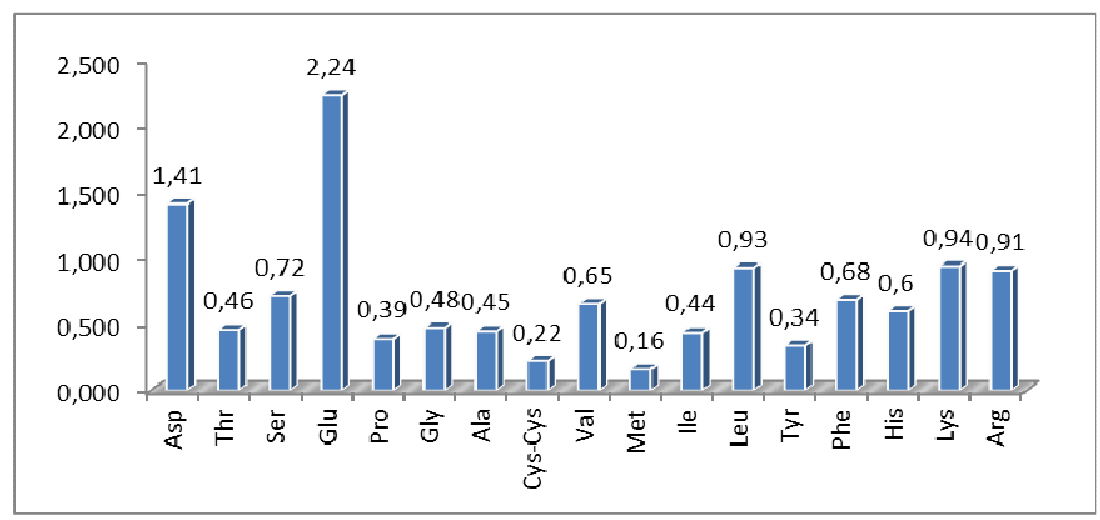

Figure 1: Teneurs en acides aminés des protéines de la Vamine, exprimées en milligramme par cent grammes de matière fraîche(MF).

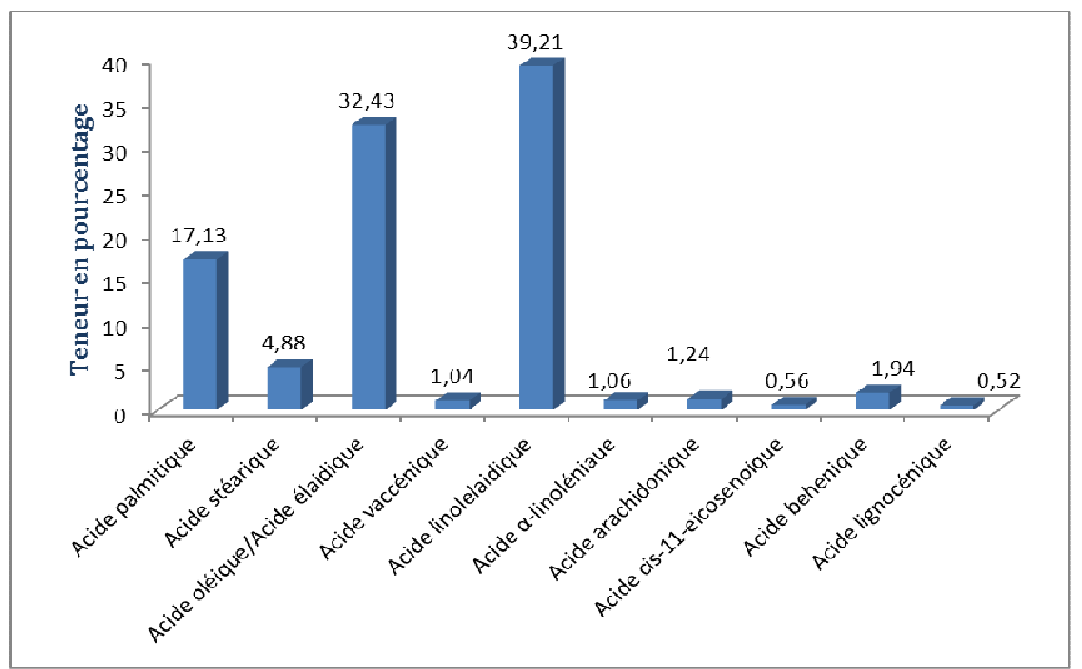

Figure 2: Teneurs en acides gras de la farine Vamine exprimées en pourcentage de la quantité totale des acides gras saturés et insaturés.

Tableau 1: Teneurs en acides aminés essentiels de la Vamine comparé à celles recommandées pour un aliment de complément.

\begin{tabular}{|c|c|c|}
\hline $\begin{array}{l}\text { Acides aminés } \\
\text { essentiels }\end{array}$ & $\begin{array}{l}\text { Vamine en mg } \\
/ 100 \mathrm{Kcal}\end{array}$ & Normes FAO /OMS \\
\hline Thréonine & 115 & 77 \\
\hline Valine & 163,5 & 99 \\
\hline Méthionine & 39,25 & 23 \\
\hline Isoleucine & 109,5 & 83 \\
\hline Phénylalanine & 171 & 75 \\
\hline Histidine & 149,5 & 45 \\
\hline Lysine & 235,25 & 119 \\
\hline
\end{tabular}




\section{DISCUSSION}

La Vamine est produite à partir du voandzou, de l'arachide, du maïs, du manioc doux, et de l'igname. Les légumineuses et le maïs sont ceux qui apportent le plus d'acides aminés. Cependant, les teneurs en acides aminés essentiels de la Vamine sont plus élevées en comparaison à celles correspondant aux normes FAO/OMS, 2009 comme le montre le Tableau1.

La Vamine a des concentrations élevées particulièrement en lysine, en histidine, en phénylalanine et en méthionine, qui ont des rôles physiologiques importants dans l'organisme croissant de l'enfant. La présence des légumineuses comme le voandzou, le niébé et l'arachide apporte la lysine tandis que le maïs apporte la méthionine. Le conditionnement des farines en quantités consommables pour l'enfant tiendra sans doute compte des normes recommandées. Il est à noter aussi la présence de tous les acides aminés essentiels, à l'exception du tryptophane que nous n'avons pas pu déceler probablement parce qu'il est très sensible aux acides et souvent détruit au cours de l'hydrolyse acide des protéines (Mbemba, 2013).

De tous les aliments qui composent la Vamine, l'arachide et le maïs contiennent respectivement des concentrations importantes en acides linoléique et arachidonique appelés omega6. Cent grammes d'huile d'arachides contiennent $25 \mathrm{~g}$ d'oméga 6 et $100 \mathrm{~g}$ d'huile de maïs contiennent 53 grammes d'oméga6; cependant les deux huiles ont des concentrations inférieures à $1 \mathrm{~g}$ d'oméga3 pour $100 \mathrm{~g}$ d'huile. Si l'acide arachidonique se trouve à $1,2 \%$, et l'acide alpha linolénique à $1,0 \%$, l'acide linoléique, qui se trouve de 3 à $5 \%$ dans l'arachide, se révèle sous la forme trans dans nos résultats. Des études épidémiologiques rapportent qu'il y aurait une relation certaine entre la consommation d'acides gras trans dérivés des huiles hydrogénées et les maladies cardiovasculaires (Mbemba, 2013). Cependant, il est peu probable que le métabolisme des acides gras essentiels soit affecté par les acides gras trans lorsqu'ils sont ingérés selon les quantités recommandés, soit 1 à $2 \%$ de l'apport énergétique total. Néanmoins, il importe de savoir si cette forme est acquise lors de la préparation de l'aliment (Murray et al., 2010).

$\mathrm{Au}$ regard des normes $\mathrm{FAO} / \mathrm{OMS}$, 2009 qui recommandent 50 à $100 \mathrm{mg} / \mathrm{kg}$ d'acide linolénique pour un aliment de complément, la Vamine en renferme $265 \mathrm{mg}$, et son conditionnement répondra sans doute à la norme ci-dessus. La consommation de la Vamine par le nourrisson garantira le développement de sa fonction cérébrale, du processus visuel, des réponses immunitaires et inflammatoires, et la production de molécules dites « hormone-like » (Briend, 2009).

\section{Conclusion}

La période de l'alimentation de complément est une période critique, surtout dans les pays pauvres où les familles ont un accès limité aux produits d'origine animale. $\mathrm{La}$ diversification de l'alimentation, et l'inclusion de produits d'origine animale ou végétale sont essentiels pour couvrir la totalité des besoins nutritionnels de l'enfant. L'étude des teneurs en acides aminés essentiels de la Vamine montre des valeurs satisfaisantes par rapport aux normes FAO. A l'exception du tryptophane qui n'a pas été dosé car très sensible aux acides et souvent détruit au cours de l'hydrolyse acide des protéines, tous les acides aminés particulièrement les acides aminés essentiels ont été rencontrés dans la Vamine.

Quant aux acides gras essentiels, la présence de l'acide $\alpha$-linolénique a une valeur de $265 \mathrm{mg} / 100 \mathrm{kcal}$, et celle de l'acide arachidonique dont le métabolisme donne les différents composés eicosanoides confirment la qualité nutritionnelle de la Vamine comme aliment de complément. Cependant, la forme trans de l'acide linoléique dans la Vamine reste à clarifier au regard des dangers qu'elle représente pour la santé de l'enfant.

A ce stade, il devient important de tester la Vamine in vivo sur un modèle animal pour en tirer des conclusions sur la croissance des enfants en RDC afin d'évaluer les risques. 


\section{REFERENCES}

Beaufrère $\mathrm{B}$, Briend $\mathrm{A}$, Ghisolfi $\mathrm{J}$, Goulet $\mathrm{O}$, Putet G, Rieu D. 2001. Nourrissons, enfants et adolescents. In AFSSA, CNERNA-CNRS. Apports nutritionnels conseillés pour la population française 3ed Tec et Doc ed Londres-Paris New York, p 255-291. Bulletin de la Société Royale des Sciences de Liège, 80: 748 - 758

Bouwer ID, Traore A, Trêche S. 2000. Activités du 2ème atelier international. voies alimentaires d'amélioration des situations nutritionnelles en Afrique de l'ouest. Ouagadougou, Burkina Faso.745-773.

Briend A. 2000. Prévention et traitement de la malnutrition. Guide pratique. Edition de l'Orstom Institut Français de recherche Scientifique pour le Développement en Coopération, 6-7.

Briend A. 2009. L'alimentation de complément: Recommandations actuelles, Institut de Recherche pour le développement, Marseille, France. Méd Trop; 69: 298-302.

FAO/OMS. 2009. Programme mixte FAO/OMS sur les normes alimentaires. Commission du Codex Alimentarius, 32ème session Rome (Italie), Rapport de la 30ème session du comité du codex sur la nutrition et les aliments diététiques ou de régime. Le Cap (Afrique du Sud) 3-7 Novembre 2008. $1-223$
Francisca JG. 2014. Intestin grêle. Le sevrage de l'allaitement. Comité de la pédiatrie communautaire, Société canadienne de pédiatrie. Paediatrics \& Child Health, 9(4): 259-263.

Mbemba F. 2013. Aliments et denrées alimentaires traditionnels du Bandundu en R.D. Congo. Répertoire et composition en nutriments, 317.

Mouquet-rivier C. 2006. L'alimentation de complément de jeunes enfants au Burkina Faso, journée portes ouvertes du «CIRD», centre IRD de Ouagadougou. Présentation de power point, 1-59.

Murray Bender A, Bothan M, Kennelly, Rodwell W, Anthony. 2010. Biochimie de Harper (28 édn). De Boeck: Bruxelles.

OPS (Organisation Panaméricaine de la Santé). 2003. Principes Directeurs pour l'Alimentation de complément de l'enfant allaité au sein. Alimentation et Nutrition 8, OPS.

Pamplona RG. 2000. Croquez la Vie. Ed. Vie te Santé : Paris ; 19-31.

Salle B. 2009. Alimentation du nouveau né et $\mathrm{du}$ nourrisson. Bulletin Académie Nationale de Médecine, 193(2): 431446. 